\title{
Development of Nicardipine Prolonged-Release Implants After Clipping for Preventing Cerebral Vasospasm: From Laboratory to Clinical Trial
}

\author{
Hidetoshi Kasuya*
}

Department of Neurosurgery, Medical Center East, Tokyo Women's Medical University, 2-1-10 Nishiogu, Arakawa-ku, Tokyo 116-8567, Japan

\begin{abstract}
Currently, there are no drugs supported by sufficient evidence of efficacy for cerebral vasospasm in patients with subarachnoid hemorrhage, despite abundant evidence of anti-vasospasm drugs at an experimental level. We have developed a drug-delivery system using a vasodilating drug that can be implanted intracranially at the time of surgery for aneurysm clipping, without systemic side effects or side effects associated with long-term intrathecal drug administration through indwelling catheters. We started our project on 1994 for making slowly-releasing drug-delivery system in vitro, because cerebral vasospasm occurs 4-14 days following subarachnoid hemorrhage. A rod-shaped pellet (1 mm in diameter, $10 \mathrm{~mm}$ in length, and containing $1 \mathrm{mg}$ of nicardipine) for animal study was prepared by heat compression. Release curve from the pellets was adjusted similar to the time course of cerebral vasospasm by changing the combination of molecular weight, lactic acid ratio of copoly (lactic/glycolic acid) and nicardipine. We presented the efficacy and safety of this drug delivery system using both canine double hemorrhage and clot placement model. The mean concentration of nicardipine in the clots was $1.5 \times 10^{-4} \mathrm{~mol} / \mathrm{L}$ on Day 7 and $5.1 \times 10^{-6} \mathrm{~mol} / \mathrm{L}$ on Day 14 . This drug delivery system can prevent vasospasm significantly in dogs, while maintaining an appropriate concentration of nicardipine in the clot adjacent to the arteries, since maximal relaxation is achieved by $10^{-6} \mathrm{~mol} / \mathrm{L}$ of nicardipine. Since October 1999 , nicardipine pellets (NPs) (2 $\mathrm{mm}$ in diameter, $10 \mathrm{~mm}$ in length, and containing $4 \mathrm{mg}$ of nicardipine) have been used to prevent vasospasm in patients with SAH. A frontotemporal craniotomy and a midline frontal craniotomy were performed for aneurysms in the internal carotid artery (ICA), middle cerebral artery (MCA), basilar artery, anterior communicating artery, and distal anterior cerebral artery (ACA). NPs were placed in the cistern of the ICA, the MCA, and/or the ACA, where thick clots existed, and, therefore, vasospasm related to delayed ischemic neurological deficits (DIND) was highly probable. The number of pellets and the location of the placement depended on the amount and site of the subarachnoid clot in the preoperative CT scans, the operative field, and the craniotomy. Cerebral vasospasm was assessed by DIND, cerebral angiography performed on Days 7 to 12 and CT scan. Vasospasm was completely prevented in the arteries in cisterns with thick clots, where vasospasm was highly expected, by placing NPs adjacent to the arteries during surgery. In the first 100 patients treated with NPs, the ratio of DIND, severe angiographical vasospasm and cerebral infarctions were $7 \%, 11 \%$, and 5\%, respectively. No complications were experienced. Thirty-two patients with severe SAH and undergoing aneurysm clipping were included into the single center, randomized, double-blind trial in Germany. The incidence of angiographic vasospasm in proximal vessel segments was significantly reduced after implantation of NPs (73\% control versus 7\% NPs). Implantation of NPs also improves clinical outcome of SAH patients. Implantation of NPs reduces the incidence of cerebral vasospasm and delayed ischemic deficits and improves clinical outcome after subarachnoid hemorrhage.
\end{abstract}

Keywords: Drug delivery, nicardipine, subarachnoid hemorrhage, vasospasm.

\section{INTRODUCTION}

Despite extensive investigative efforts, the pathogenesis and pathophysiology of delayed cerebral vasospasm after aneurismal subarachnoid hemorrhage (SAH) remain far from clear. Vasospasm continues to be one of the primary causes of mortality and neurological morbidity and an important cause of cerebral ischemia and stroke, despite the establishment of early surgical obliteration of aneurysm. Although there have been numerous reports describing the prevention of DIND such as intrathecal administration of urokinase, cisternal irrigation with drainage, endovascular

*Address correspondence to this author at the Department of Neurosurgery, Medical Center East, Tokyo Women's Medical University, 2-1-10 Nishiogu, Arakawa-ku, Tokyo 116-8567, Japan; Tel: +81-3-3810-1111; Fax: +81-3-3819-8680; E-mail: kasuyane@dnh.twmu.ac.jp,

3z7n3v@bma.biglobe.ne.jp treatment, head shaking, arterial injection of vasodilatory drug [1], most of them are complicated even if they are effective.

We have developed a drug-delivery system using a vasodilating drug that can be implanted intracranially at the time of surgery for aneurysm clipping and have been using the drug to SAH patients since 1994. The current report presents how we have been developing this drug delivery system from laboratory to clinical trial.

\section{PRELIMINARY STUDY USING PAPAVERINE}

A drug delivery system using copoly (lactic/glycolic acid) was developed for the intracranial administration of papaverine [2]. A rod-shaped implant prepared by a heat compression method was tested to determine its efficacy in preventing cerebral vasospasm in dogs. Sixteen dogs were 
randomly assigned to one of two groups, i.e., placebo or papaverine. Control angiography was performed, followed by right craniectomy and the induction of subarachnoid hemorrhage by the placement of a clot in the Sylvian fissure. Two pellets, containing either $25 \mathrm{mg}$ of papaverine or no papaverine, were placed in the cistern. In in vitro studies, $56 \%$ of the actual papaverine loading was released in the first 4 days and 78\% within 8 days. On Day 7, angiography was repeated and the animals were killed. A similar experiment using low-dose pellets containing $5 \mathrm{mg}$ of papaverine, half of which was released within 7 days, was performed with 16 mongrel dogs.

There were significant differences between the papaverine- and placebo-treated groups in the reductions of vessel diameters of the internal carotid, middle cerebral, and anterior cerebral arteries on the clot side. The mean concentration of papaverine in the clot was $4.5 \times 10^{-4} \mathrm{~mol} / \mathrm{L}$. The low-dose pellet failed to prevent cerebral vasospasm, although the mean concentration of papaverine in the clot was $2.3 \times 10^{-5} \mathrm{~mol} / \mathrm{L}$. A prolonged-release preparation of papaverine implanted intracranially at the time of surgery prevented vasospasm significantly while maintaining an appropriate concentration of papaverine in the cistern. However, the effective dose was much higher than we expected from the data in vitro and we concluded that papaverine is not practical vasodilating drug for clinical use.

\section{IN VITRO STUDY}

A rod-shaped pellet $(2 \mathrm{~mm}$ in diameter, $10 \mathrm{~mm}$ in length, containing $4 \mathrm{mg}$ of nicardipine, for animal model $1 \mathrm{~mm}$ in diameter, $10 \mathrm{~mm}$ in length, containing $1 \mathrm{mg}$ of nicardipine) was prepared by heat compression. Copoly (lactic/glycolic acid) (PLGA) (PLG1600ML; molecular weight 4000, lactic acid ratio 0.5) was purchased from Taki Co. (Kakogawa, Kobe, Japan). A mixture of PLGA (900 mg) and nicardipine free base $(100 \mathrm{mg})$ was dissolved in dichloromethane (10 $\mathrm{ml}$ ). The dichloromethane was evaporated with a rotary evaporator, and the resultant mass was dried further under vacuum. The dried powder $(40 \mathrm{mg})$ was charged into a Teflon tube (2-mm inner diameter). The tube was set in a stainless steel cylinder kept at $35-40^{\circ} \mathrm{C}$. A pressure of 100 $\mathrm{kg} / \mathrm{cm}^{2}$ was applied between the upper and lower stainless steel dies. The compressed pellet was sterilized by $\gamma$-ray (Nippon Shosha Service, Tokai, Ibaraki, Japan). Nicardipine free base was prepared as follows: Nicardipine $\mathrm{HCl}$ (Sigma Chemical Co., St. Louis, MO) was dissolved in water. $\mathrm{NaOH}$ $(5 \mathrm{~N})$ was added to the solution to shift the $\mathrm{pH}$ above 10 . The nicardipine free base was extracted with dichloromethane [37].

The release of nicardipine from the pellet was examined

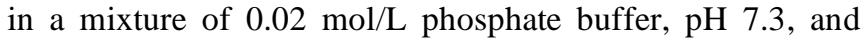
saline $(1: 1, \mathrm{v} / \mathrm{v})$ in a flask. The flask was shaken in a waterbath (37[degrees]C) at a frequency of 20 strokes/min. Five milliliters of the release medium were withdrawn periodically and replaced with an equivalent volume of fresh buffer. The amount of papaverine released was analyzed by high performance liquid chromatography (Hitachi L6000; Hitachi, Tokyo, Japan), under the following conditions: column, YMC-Pack ODS AM-312 (150 mm x 6.0-mm inner diameter); column temperature, 40 [degrees]C; mobile phase, $10 \mathrm{mmol} / \mathrm{L} \mathrm{KH} 2 \mathrm{PO} 4 /$ acetonitrile (6:4); flow rate, 1.5 $\mathrm{ml} / \mathrm{min}$; detection, ultraviolet absorbance at $240 \mathrm{~nm}$; internal standard, pyrilamine (obtained from Sigma Chemical Co., St. Louis, MO).

Release curve from the pellets was adjusted similar to the time course of cerebral vasospasm by changing the combination of molecular weight and lactic acid ratio of Copoly (lactic/glycolic acid) and nicardipine (Fig. (1)).

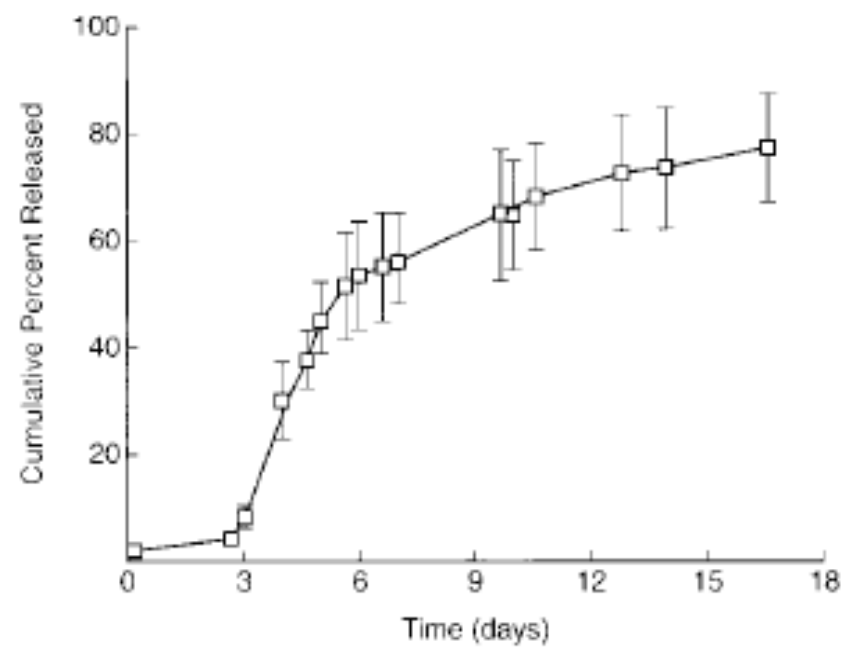

Fig. (1). Release of nicardipine from prolonged-release pellets for human in vitro. The release of nicardipine from pellets was calculated as the percentage of the amount released at time, compared with the actual drug loading (mean $\pm \mathrm{SD})(\mathrm{n}=4)$.

\section{ANIMAL MODEL}

\section{Canine Clot Placement Model}

The purpose of this study was to determine the efficacy of nicardipine prolonged-release implants for preventing vasospasm in a canine SAH model in a dose-escalating placebo-controlled blind fashion. Drug-release kinetics of copoly (lactic/glycolic acid) pellet containing nicardipine was evaluated in vitro. In vivo, 18 dogs were randomly assigned to one of three groups, i.e. placebo, low-dose $(0.8$ $\mathrm{mg}$ ), or high-dose $(8 \mathrm{mg}$ ) nicardipine. Angiography was performed, followed by right craniectomy, the induction of subarachnoid hemorrhage, and the placement of the pellets in the Sylvian fissure. On Day 7 and Day 14, the angiography was repeated. In the first four days, $61.9 \%$ of the actual nicardipine loaded was released and within 10 days, $96 \%$. The average percent reductions of vessel diameters in the middle cerebral artery on Day 7 were $43 \%, 14 \%$ and $7 \%$ in the placebo, low-dose, and high-dose groups, respectively ( $\mathrm{p}$ $=0.0319)$ (Fig. (2)). The mean concentration of nicardipine in the clots on Day 14 was $9.7 \times 10^{-7} \mathrm{~mol} / \mathrm{L}$ and $5.1 \times 10^{-6}$ $\mathrm{mol} / \mathrm{L}$ in the low-dose and high-dose group, respectively. This drug delivery system prevented vasospasm in dogs significantly even at low dose, while maintaining an appropriate concentration of nicardipine in the clot adjacent to the arteries [3, 4].

\section{Canine Double Hemorrhage Model}

Ten dogs were assigned to two groups: double hemorrhage group and double hemorrhage group treated with implants. Vertebral angiography and arterial blood 


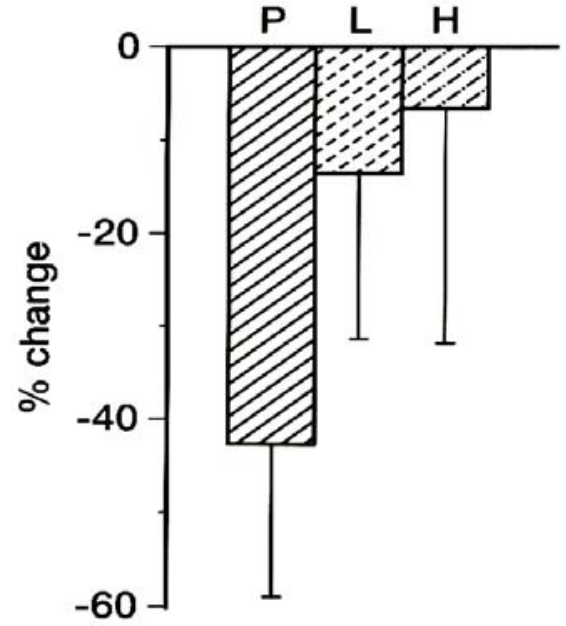

Fig. (2). Effect of nicardipine prolonged-release pellets on the diameters of middle cerebral arteries of the clot side in canine clot placement model. Bars, percentage (mean $+\mathrm{SD}$ ) reduction on Day7, from control values (Day 0). There were significant differences in the diameters between placebo $(\mathrm{P})$, low dose $(\mathrm{L})$, and high dose $(\mathrm{H})$ groups. There were significant differences between $\mathrm{P}$ and $\mathrm{L}$, between $\mathrm{P}$ and $\mathrm{H}$, but not between $\mathrm{L}$ and $\mathrm{H}$.

injection into the cisterna magna were performed, followed by midline suboccipital craniectomy and laminectomy of the atlas and placement of nicardipine implants in the cisterna magna. On day 2, arterial blood injection into the cisterna magna was repeated (double hemorrhage model). On day 7 , vertebral angiography was repeated. The animals were then sacrificed and the brain and blood clot were taken out. All the animals involved in both groups had been clinically well. Though 5 animals of the control group showed severe vasospasm, no vasospasm was observed in 3 animals and only very mild vasospasm in 2 of the nicardipine-treated group. There was a statistically significant difference in diameter between the two groups $(0.5 \mathrm{~mm}$ vs $1.1 \mathrm{~mm}, \mathrm{p}=$ 0.009) (Fig. (3)). Histological examination showed no specific changes related to implants. Neither clinical symptoms related to implants nor specific histological changes were observed (e.g. hypotension, seizure). These results suggested that nicardipine-prolonged release preparation is safe as well as effective for cerebral vasospasm [5].

\section{CLINICAL TRIAL}

\section{The First Consecutive 100 Patients}

Nicardipine prolonged-release implants (NPRIs) have been used to prevent vasospasm in patients with SAH since October 1999. The study analyzed the efficacy and safety of NPRIs in 100 patients with SAH and thick subarachnoid clot (mainly Fisher group 3) treated with NPRIs (diameter $2 \mathrm{~mm}$, length $10 \mathrm{~mm}$, containing $4 \mathrm{mg}$ of nicardipine) during surgery after clipping of the aneurysm. The number and location of pellets depended on the amount and site of the subarachnoid clot on preoperative computed tomography and on the type of craniotomy. Two to 12 pellets were implanted in the cisterns of the internal carotid artery, middle cerebral artery, and/or anterior cerebral artery, where thick clots were present and vasospasm related to delayed ischemic

\section{diameter of basilar artery}

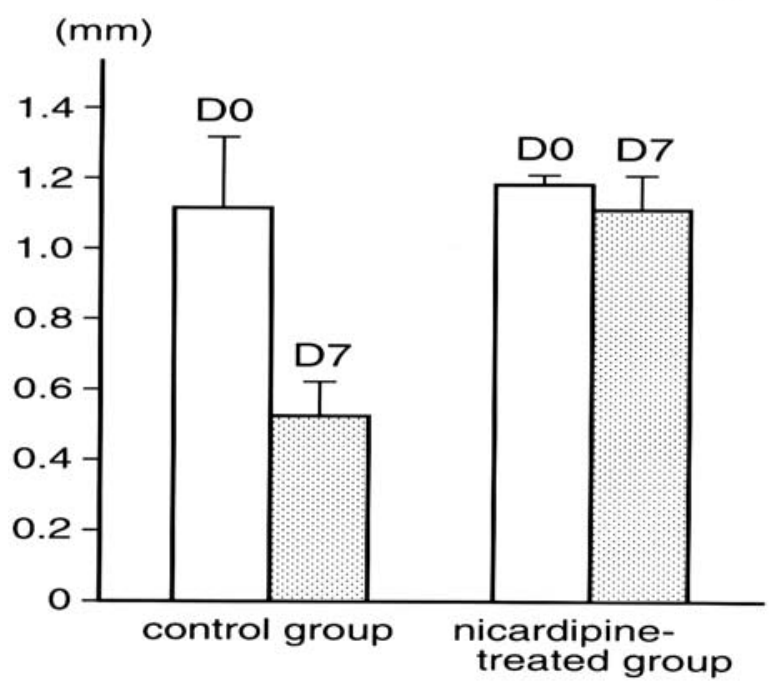

Fig. (3). Effect of nicardipine prolonged-release pellets on the diameters of basilar arteries in canine double hemorrhage model. The bars represent the average diameter of basilar arteries $( \pm S D)$. There was a significant difference in the diameter on day 7 between control and nicardipine-treated groups. D0; day 0, D7; day7.

neurological deficit (DIND) was highly likely. Only seven patients developed DIND and five patients suffered cerebral infarction. Angiography performed on days 7-12 revealed no vasospasm in any of the arteries close to the site of NPRI placement. NPRI placement can completely prevent vasospasm in arteries within the cisterns containing thick clots, but is less effective in remote locations [6-12] (Table 1).

Table 1. The Degree of Vasospasm in the First Consecutive 100 Patients with SAH Treated with Nicardipine Prolonged-Release Pellets

\begin{tabular}{|l|l|}
\hline Total & $\mathbf{1 0 0}$ \\
\hline \hline No or mild vasospasm on angiography & 89 \\
\hline $\begin{array}{l}\text { A severe degree of vasospasm on the arteries not adjacent to } \\
\text { pellets: the other side of craniotomy, more distal arteries }\end{array}$ & 11 \\
\hline DIND & 7 \\
\hline Infarction & 5 \\
\hline Side effects & 0 \\
\hline
\end{tabular}

\section{Single Center, Randomized, Double-Blind Trial}

Thirty-two patients with severe subarachnoid hemorrhage and undergoing aneurysm clipping were included into this single center, randomized, double-blind trial. Sixteen patients received NPRIs implanted into the basal cisterns in direct contact to the exposed proximal blood vessels; in 16 control patients, the basal cisterns were opened and washed out only without leaving implants. Angiography was performed preoperatively and at day $8+/-1$. Computed tomography imaging was analyzed for the incidence of territorial infarcts unrelated to surgery. Patient outcome was 
Table 2. Severity of Proximal Angiographic Vasospasm

\begin{tabular}{|l|l|l|l|}
\hline & Control & NP & p \\
\hline \hline C1 & $87 \pm 19$ & $113 \pm 19$ & 0.0021 \\
\hline C1 contra & $85 \pm 28$ & $115 \pm 18$ & 0.0024 \\
\hline M1 & $75 \pm 28$ & $110 \pm 25$ & \\
\hline M1 contra & $75 \pm 35$ & $117 \pm 26$ & 0.0004 \\
\hline A1 & $62 \pm 25$ & $116 \pm 39$ & 0.0152 \\
\hline A1 contra & $79 \pm 35$ & $125 \pm 34$ & $117 \pm 18$ \\
\hline P1 & $88 \pm 26$ & $128 \pm 14$ & \\
\hline P1 contra & $81 \pm 22$ & \\
\hline
\end{tabular}

The given data represent the calculated relative values (\%) of the resulting vessel diameter on the day 8 angiogram compared to baseline.

assessed using the modified Rankin and National Institute of Health Stroke scales. The incidence of angiographic vasospasm in proximal vessel segments was significantly reduced after implantation of NPRIs (73\% control versus $7 \%$ NPRIs). Significant differences occurred also for the majority of distal vessel segments. Computed tomography scans revealed a lower incidence of delayed ischemic lesions (47\% control versus 14\% NPRIs). The NPRI group demonstrated more favorable modified Rankin and National Institute of Health Stroke scales as well as a significantly lower incidence of deaths (38\% control versus $6 \%$ NPRIs). Implantation of NPRIs reduces the incidence of cerebral vasospasm and delayed ischemic deficits and improves clinical outcome after severe subarachnoid hemorrhage [13, 14] (Table 2).

\section{Multicenter Cooperative Study in Tokyo}

We started a multicenter cooperative study on Jan 1, 2007, and 136 patients in six hospitals were enrolled to this trial in two years. The incidence of cerebral vasospasm and outcome were examined in these patients. The patients with SAH were treated with NP (nicardipine pellet) during surgery after clipping of their aneurysms. The study included 87 female patients, 38 over 70 years old, 34 in grades 4 and 5 , and 46 of Fisher group 2 or 4 . Aneurysms were located on anterior circulation in 133, posterior in 3. All patients were treated with Fasudil hydrochloride except for 3. Two to 12 pellets were implanted in the cistern where thick clots existed and vasospasm was highly likely. Delayed ischemic neurological deficits (DIND), angiographical vasospasm and cerebral infarctions were seen in 11 of $134(8.2 \%), 32$ of 130 patients $(24.6 \%)$, and 16 of $129(12.4 \%)$, respectively. No complications were experienced. Independent rate at 3 months was $78 \%$. The incidence of cerebral vasospasm in this multicenter trial is similar to that of our first trial performed in a single center [15] (Table 3).

\section{DISCUSSION}

Currently, there are no drugs supported by sufficient evidence of efficacy for cerebral vasospasm in patients with subarachnoid hemorrhage, despite abundant evidence of antivasospasm drugs at an experimental level. The problem could not be solved by developing new drugs but by developing methods to maintain an appropriate concentration of the drug in the target cerebral artery and its surrounding environment. We consider that this could not be achieved by systemic administration of drugs but rather by local treatment. It is, however, also difficult to maintain an effective concentration by intrathecal administration of vasodilating drugs, if the agent is water-soluble, because CSF circulation dilutes and washes out the drug. We think that the success of our drug-delivery system is influenced by choosing the drug: nicardipine [9].

Vasospasm was completely prevented in arteries in cisterns with thick clots, where vasospasm is highly expected, by placing pellets adjacent to the arteries during surgery. Fewer efficacies were found for arteries remote from the placement of pellets [7]. This was expected from our in vitro findings of high lipophilicity of nicardipine. Nicardipine was probably adsorbed to the clot and arterial tissue near the pellets because of its high lipophilicity and did not affect the remote arteries, since nicardipine was not detected in any cerebrospinal fluid samples in our experimental model [3-5]. We may find ideal drugs with less lipophilicity that work in the very local region and more remote arteries, or a more effective cocktail of drugs with different characteristics may be developed.

Since the literature describes DIND to be much more common as well as the associated poor outcome, our results suggest that the application of NP to SAH patients may prevent vasospasm related cerebral infarctions and therefore avoid an unfavorable outcome. The efficacy was proven by a randomized double-blinded controlled trial performed in Germany [13]. This drug-delivery system offers a promising approach for preventing vasospasm when a craniotomy is performed as part of the aneurysm treatment. However, the application of NP has its limitations: such as for the arteries on the contralateral side of the craniotomy, or the more distal arteries. We were not able to use these pellets for patients that were treated by coiling. This problem may be solved by developing a new drug delivery system which allows the maintainance of an appropriate concentration of nicardipine in the target artery, since local application of nicardipine is able to completely prevent vasospasm [9].

\section{CONCLUSION}

We started our project on 1994 for making slowlyreleasing drug-delivery system in vitro, because cerebral 
Table 3. Cerebral Vasospasm and Outcome in 136 Patients Treated with Nicardipine Pellets

\begin{tabular}{|l|l|l|}
\hline & & Number of Patients \\
\hline \hline DIND* & & $11 / 134$ \\
\hline Angiographical vasospasm (moderate, severe) & & $32 / 130$ \\
\hline Low density area on CT & & $16 / 129$ \\
\hline Hydrocephaus & & 43 \\
\hline Outcome (Glasgow) at 3 months & Good & 85 \\
\hline & Moderate disability & 21 \\
\hline & Severely disability & 15 \\
\hline Total & Persistent vegetative & 6 \\
\hline & Dead & 9 \\
\hline
\end{tabular}

*DIND: delayed ischemic neurological deficits.

vasospasm occurs 4-14 days following subarachnoid hemorrhage. We presented the efficacy and safety of this drug delivery system using both canine double hemorrhage and clot placement model. Since October 1999, nicardipine pellets (NPs) (2 $\mathrm{mm}$ in diameter, $10 \mathrm{~mm}$ in length, and containing $4 \mathrm{mg}$ of nicardipine) have been used to prevent vasospasm in patients with SAH. Implantation of NPs reduces the incidence of cerebral vasospasm and delayed ischemic deficits and improves clinical outcome after subarachnoid hemorrhage.

\section{REFERENCES}

[1] Keyrouz, S.G.; Diringer, M.N. Clinical review: Prevention and therapy of vasospasm in subarachnoid hemorrhage. Crit. Care, 2007, 11, 220 .

[2] Shiokawa, K.; Kasuya, H.; Miyajima, M.; Izawa, M.; Takakura, K. Prophylactic effect of papaverine prolonged-release pellet on cerebral vasospasm in dogs. Neurosurgery, 1998, 42, 109-116.

[3] Kawashima, A.; Kasuya, H.; Shiokawa, K.; Miyajima, M.; Izawa, M.; Takakura, K. Efficacy of nicardipine prolonged-release pellet on cerebral vasospasm in dogs. No Shinkei Geka, 1988, 26, 37-43.

[4] Kawashima, A.; Kasuya, H.; Sasahara, A.; Miyajima, M.; Izawa, M.; Hori, T. Prevention of cerebral vasospasm by nicardipine prolonged-release implants in dogs. Neurol. Res., 2000, 22, 634641.

[5] Sasahara, A.; Kasuya, H.; Kawashima, A.; Aihara, Y.; Izawa, M.; Hori, T. The efficacy and safety of the nicardipine prolongedrelease implant in a canine double hemorrhage model. No Shinkei Geka, 2000, 28, 1071-1075.

[6] Kasuya, H.; Kawashima, A.; Sasahara, A.; Onda, H.; Hori, T. Development of nicardipine prolonged-release implants for preventing vasospasm. Acta. Neurochir. Suppl., 2001, 77, 217-220.
[7] Kasuya, H.; Onda, H.; Takeshita, M.; Okada, Y.; Hori, T. Efficacy and safety of nicardipine prolonged-release implants for preventing vasospasm in humans. Stroke, 2002, 33, 1011-1015.

[8] Kasuya, H.; Onda, H.; Sasahara, A.; Takeshita, M.; Hori, T. Application of nicardipine prolonged-release implants: Analysis of 97 consecutive patients with acute subarachnoid hemorrhage. Neurosurgery, 2005, 56, 895-905.

[9] Kasuya, H. Application of nicardipine prolonged-release implants: Analysis of 97 consecutive patients with acute subarachnoid hemorrhage. Neurosurgery, 2006, 58, E799.

[10] Krischek, B.; Kasuya, H.; Onda, H.; Hori, H. Clinical trial of nicardipine prolonged-release implants for preventing vasospasm: Analysis of 100 consecutive patients. Neurol. Med. Chir. (Tokyo), 2007, 47, 389-396.

[11] Kasuya, H.; Onda, H.; Krischek, B.; Hori, T. Cerebral vasospasm following subarachnoid hemorrhage is completely prevented by Ltype calcium channel antagonist in human. Acta Neurochir. Suppl., 2008, 104, 109-114.

[12] Kasuya, H.; Krischek, B. Clinical trial of nicardipine prolongedrelease implants for preventing vasospasm: Analysis of 100 consecutive patients. Neurol. Med. Chir. (Tokyo), 2008, 48, 147148.

[13] Barth, M.; Capelle, H.H.; Weidauer, S.; Weiss, C.; Münch, E.; Thomé, C.; Luecke, T.; Schmiedek, P.; Kasuya, H.; Vajkoczy, P. Effect of nicardipine prolonged-release implants on cerebral vasospasm and clinical outcome following severe aneurysmal subarachnoid hemorrhage - a prospective, randomized, doubleblinded phase IIa study. Stroke, 2007, 38, 330-336.

[14] Barth, M.; Thome, C.; Schmiedek, P.; Weiss, C.; Kasuya, H.; Vajkoczy, P. Characterization of functional and quality of life in patients following SAH with and without nicardipine prolonged release pellets. J. Neurosurg., 2009, 110, 955-960.

[15] Kasuya, H. Clinical trial of nicardipine pellets for preventing cerebral vasospasm: Multi-center cooperative study in Tokyo. Acta Neurochir. Suppl., (in press).

(C) Hidetoshi Kasuya; Licensee Bentham Open .

This is an open access article licensed under the terms of the Creative Commons Attribution Non-Commercial License (http://creativecommons.org/licenses/by-nc/3.0/) which permits unrestricted, non-commercial use, distribution and reproduction in any medium, provided the work is properly cited. 